\title{
Good Mothering Before Birth: Measuring Attachment and Ultrasound as an Affective Technology
}

\author{
JENNIFER DENBOW \\ CALIFORNiA POLyTECHNiC STATE UNIVERSITY, SAN LUIS OBISPO
}

\begin{abstract}
The idea that fetal ultrasound is useful for promoting a pregnant woman's emotional attachment to her fetus is commonplace in the United States. While STS scholars have examined many facets of ultrasound, scholars have not analyzed the medical construction of ultrasound as an affective technology. This article fills that gap by bringing feminist STS and affect studies together to examine medical understandings of fetal ultrasound's emotional utility. The project interprets a unique archive of published medical research on measuring maternal-fetal bonding and using ultrasound to promote that bonding. My discourse analysis shows that this medical research defines "optimal bonding" in a way that reflects the norms of intensive mothering. I argue that this medical research contributes to the creation of a new, presumably high-risk population of "sub-optimal bonders." The research I examine also suggests that medical professionals may be able to use the technological fix of ultrasound to manage this new population's emotions and behaviors. In the process, medical experts individualize the risks of infant well-being and locate those risks in women's emotional state.
\end{abstract}

\section{Keywords}

STS; feminist studies; affect theory; reproduction

\section{Good Mothering Before Birth: Measuring Attachment and Ultrasound as an Affective Technology}

Ultrasound requirements ensure informed choice because they allow a woman to see her unborn child as he or she really is, by seeing his or her form and face on a screen ... [M]edical evidence indicates that women feel bonded to their children after seeing them on the ultrasound screen. Once that bond is established, researchers argue, a woman no longer feels ambivalent toward her pregnancy and actually begins to feel invested in her unborn child.

-Americans United for Life 2

"Jennifer Denbow, Email: jdenbow@calpoly.edu
"From "Women's Ultrasound Right to Know Act" (2011). Accessed 20 February 2018:
http://www.aul.org/defending-life-2011-model-legislation-abortion/

Copyright (C) 2019 (Jennifer Denbow). Licensed under the Creative Commons Attribution Non-commercial No Derivatives (by-nc-nd). Available at estsjournal.org. 
The idea that fetal ultrasound helps facilitate a pregnant woman's attachment to her fetus is now a fairly commonplace idea in the United States and many other developed countries (see Kukla 2008). In fact, this idea has made its way into laws in the US. The above quote is from model legislation for a "Women's Ultrasound Right to Know Act" drafted by Americans United for Life (AUL), an influential pro-life legal and advocacy organization. Fourteen states have adopted preabortion ultrasound mandates similar to those in the AUL guide (Guttmacher 2018). These laws are premised on the notion that ultrasound promotes a woman's bond to her fetus and will thus dissuade her from abortion. When ultrasound was initially used during pregnancy, however, the focus of medical researchers and practitioners was on what it allowed obstetricians to see and do (Oakley 1984). This article examines how medical researchers primarily in the Global North came to view fetal ultrasound as not just a diagnostic tool, but also as a tool for managing pregnant women's affect. An important component of this research is the measurement of maternal-fetal attachment and, as explained below, this article focuses on this neglected area in science and technology studies (STS) research on reproduction. In doing so, this article brings affect studies and STS together and thus intervenes in existing STS literature on reproduction, which rarely considers the role of affect. Through analysis of a unique archive, I show how medicine and technology may play a role in keeping emotions in line with dominant norms.

Like other technologies of visualization-and as evidenced in the AUL quote abovesonograms are often viewed as unmediated, neutral, and objective pictures of reality (Joyce 2005). This perceived objectivity neglects how technical, political, and cultural forces affect the production, dissemination, and perception of these technical images (Newman 1996). Nonetheless, the development of visualization technologies has contributed to the prominence of the fetus in public discourse (Duden 1993; Newman 1996). Feminist STS scholars have in fact shown how politically motivated groups have deployed ultrasound to help constitute the fetus as an individual person separate from the pregnant woman (Franklin 1991; Haraway 1988; Petchesky 1987; Shrage 2002). Anti-abortion movements in particular rely heavily on ultrasound imagery to erase the pregnant body and promote their view of the fetus as an individual with legal rights (Petchesky 1987). Public health campaigns have also used ultrasound imagery to heighten the emotional appeal of fetuses and reinforce norms of bonding (Oaks 2000).

In addition to the above research, scholars have looked at medical contexts to understand how medical researchers and practitioners in North America and Britain understand reproduction, pregnancy, and the fetus. For example, Lynn M. Morgan (2009) has studied the history of embryology and corresponding views of the fetal body, while others have studied medical practices of fetal surgery (Casper 1998) and fetal sonography (Mitchell 2001; Palmer 2009; Taylor 2002). Julie Roberts and her colleagues have argued that some women who seek out commercial ultrasound do so in order to facilitate bonding and are influenced by discourses of good mothering (Roberts et al. 2015). Research has also uncovered the political and social contexts that have led to the development of medical ideas such as fetal alcohol syndrome (Armstrong 2008), as well as the social and legal impact of specific technologies (Rapp 2000;

${ }^{3}$ None of the research I review in this article considers the possibility of either pregnant individuals who do not identify as women or of a pregnant individual's partner being anything other than male. 
Roberts 2016). Scholars are increasingly focusing on how developments in fields like epigenetics and prenatal medicine extend women's responsibility for child well-being back in time to encompass even the period before conception (see Lappé 2016; Richardson 2015; Waggoner 2013, 2015).

This article brings the study of affect more centrally to this body of research. I examine how some medical researchers measure bonding during pregnancy and how they have come to see bonding as central to pregnant women's responsibility for fetal and infant health. ${ }^{*}$ In focusing on how a group of medical researchers came to view ultrasound as important for this bonding, this article picks up on Ann Oakley's (1984) study of fetal ultrasound in Britain. She shows that medical practitioners' desire to know about the fetus motivated the early development of fetal ultrasound. Oakley notes the turn in the early 1980s toward using fetal ultrasound to manage women's reactions to fetuses, but does not closely examine the research on ultrasound and maternal-fetal bonding. Janelle S. Taylor (2008) has also researched ultrasound and looked more closely at what she calls "the theory of ultrasound bonding." Taylor examines in depth two medical sources and points out that the theory of ultrasound bonding in general

is persuasive, not because it is grounded in sound science, but because it both emerges out of and contributes to a discourse that links visual images, via the maternal imagination, to the physical form and health of the fetus, in a manner that radically decontextualizes women's emotions and authorizes efforts to control women's behavior. $(2008,106)$

This article builds on these insights in bringing affect studies to a more expansive medical archive focused on how researchers measure maternal-fetal bonding, as well as how those measures play a role in research that investigates whether ultrasound facilitates that bonding. In doing so, the article heeds Taylor's call for scholars to "seek out and follow the trails of evidence supporting claims made in the name of science" regarding maternal-fetal bonding $(2008,115)$.

To do this and as described in detail later in the article, I traced the research on fetal ultrasound's effect on maternal-fetal bonding through editorials and peer-reviewed articles published in English-language medical journals. The bulk of studies were carried out in the US and Europe, which suggests that these discourses may be limited to the Global North.s Importantly, and as noted above, the investigation of this limited research revealed that the notion that maternal-fetal bonding can be measured through self-assessment is central to this body of research. Two common questionnaires that are used to measure this bonding are the maternal-fetal attachment scale (MFAS) and the maternal antenatal attachment scale (MAAS) (Cunen 2016). This article focuses on the scales because they are central to the study of

"Some researchers suggest that bonding and attachment are distinct and that only "bonding" should be used to describe the relation of the mother toward the fetus (see Ji et al. 2005, 473). However, much of the literature in this field uses the terms "bonding" and "attachment" interchangeably. I have followed that convention.

${ }^{5}$ For example, India outlaws the use of ultrasound for sex selection, suggesting a different political orientation to ultrasound. 
ultrasound's effect on bonding, they have not been critically analyzed to date, and they are interesting in their own right. Although the scales are central to this analysis, this article also includes a brief discussion of how these scales are implemented in the limited literature on ultrasound's effect on maternal-fetal attachment.

I bring a critical feminist science studies perspective to this topic. As Joan Fujimura has argued, such a perspective opens the conclusions of scientific studies to "multiple readings of the same data from different sociocultural perspectives or frames of reference" $(2006,50)$. While Fujimura examines the materiality of nature, I focus on the interplay of the emotional and the material. Some of the researchers under investigation here are focused on understanding the (natural, normal) emotional response of pregnant women who are confronted with a sonogram, which is often understood as revealing the true material nature of the fetus. Although affect theory is not often brought into STS scholarship, my investigation into maternal-fetal bonding centrally concerns the interplay among affect, science, and technology. Bringing affect theory to this investigation thus illuminates both how lack of right feeling, or being improperly oriented toward the fetus, becomes a medical risk or condition and also how technology becomes the means to correct affect.

I rely in particular on Sara Ahmed's feminist affect theory in The Promise of Happiness (2010) to show how technology is understood as a way of bringing pregnant individuals in line with an affective community. Members of an affective community are committed to "the same objects as the cause of happiness" (38). In this case, the affective community reflected in the studies shares a particular normative assessment of gender and reproduction. Ahmed mentions that objects like a family photograph album construct the family as a happy object (45); I extend her insight to show how a sonogram constructs the fetus as a baby and a happy object. One who has negative or ambivalent feelings about the fetus is thus, in Ahmed's terms, alienated from the affective community. Some of the medical research I studied, as well as corresponding legal developments, hold out the image of the fetus as what could dissipate or solve this alienation. The image, in its alleged truth, can ensure that what has already been ascribed as good is in fact felt as good by those most intimately connected to the object.

I also analyze the fetal body and its affective weight alongside the pregnant body to show how the fetal body's construction as an object of happiness relates to the differential construction of some pregnant bodies as optimal or sub-optimal bonders. Some of the research examined here suggests that medical professionals can use the technological fix of ultrasound to manage the emotions and thus behaviors of this new population. In the process, these experts tend to individualize the risks of infant well-being and locate those risks in women's emotional state. The research thus provides an example of how well-meaning research can simplify the causes of health inequalities in society (see Epstein 2007).

\section{Measuring Attachment}

Although historians have traced the idea that mothering consists mostly in emotional care and is primary to women's identity to late 18th century white affluent societies in North America and 
western Europe (Cott 1977; Degler 1984), mothers' emotions became an important object of medical study in the 1950s with British psychiatrist John Bowlby's attachment theory. This theory arose at a time when questions about women's mothering and work roles were widely debated, and, in Marga Vicedo's words, it "exerted a strong emotional demand on mothers and contributed to an increasing discourse of mother blame" (2013, 9-10). In the 1960s researchers began to consider attachment during the prenatal stage. This research took for granted that the fetus is a (separate) entity to which a pregnant woman can and should attach. Nurse researcher Reva Rubin examined how women attained the role of mother during pregnancy and is standardly credited with laying the groundwork for a theory of prenatal attachment. She argued that the bond between mother and infant upon birth was a consequence of the development of the maternal role before birth (Rubin 1967a, 1967b). Rubin delineated four aspects of pregnant women's transition to the maternal role: "(1) Seeking safe passage for self and baby, (2) ensuring that the baby is accepted by significant others, (3) 'binding-in', and (4) giving of herself" (Brandon 2009). "Binding-in" refers to the process by which "the idea of the child, her child, during pregnancy is [incorporated] into the woman's entire self-system: into her body image, her self-image, and her ideal image" (Rubin 1976, 372).

Research on bonding was never simply descriptive: embedded within it were tacit and sometimes explicit prescriptions for mothering (Eyer 1992). The development of the body of research on attachment coincided with an intensification of the intensive mothering ideal and with the increased medicalization of pregnancy (Oakley 1984, 2). This resulted in a focus on, in Barbara Katz Rothman's words, "the unique vulnerabilities of the fetus," which in turn led to an intensified focus on pregnant women's "'compliance,' the willingness of a patient to 'follow doctor's orders'" $(2000,59)$. Medicalization and risk culture place incredible responsibility on pregnant women for producing healthy babies (Denbow 2015; Ruhl 2002). As Joan Wolf describes, "women are confronted with an abundance of far-reaching and often highly contested knowledge about how to create optimal wombs and then to monitor their fetuses, babies, and children in order to reduce the risks of anything deemed undesirable" $(2011,75)$.

This overview of intensive mothering provides context for a discussion of tools to measure maternal-fetal bonding. The questionnaires that measure maternal-fetal attachment, which is a proxy for mother love, introduce a distinct element into the medical management of pregnancy and fetal health. Whereas Vicedo critically examined the development of attachment theory, I trace how attachment becomes something that can be measured through selfassessment. Rothman argues that quantifying things makes them "real" such that joy seems "unreal" because it is not "an observable measure for childbirth" (2000, 31). The questionnaires that are developed to measure maternal-fetal bonding extend the quantification of myriad aspects of health to the emotional sphere, making them more "real." In fact, researchers refer to these questionnaires as "instruments," invoking the language of tools that seemingly provide objective measurements. Moreover, the deepest feelings of maternal love and attachment have come to be understood, not as "sentimental" or "hysterical" (Rothman 2000, 32), but as, in the words of one researcher, "sound" (Sioda 1984, 659). 
Two common instruments for measuring maternal-fetal attachment are the maternal-fetal attachment scale (MFAS) and the maternal antenatal attachment scale (MAAS). As I show, despite differences, the instruments make similar assumptions about optimal bonding: they assume that a high intensity of preoccupation with the fetus is optimal and that pregnancy is an extraordinary event that necessitates significant lifestyle changes. The scales furthermore assume that visualizing the fetus and perceiving it as an individual are optimal. Because even ultrasound researchers who develop their own ways to measure bonding often rely on a theoretical framework similar to that undergirding the MFAS or MAAS, my analysis concentrates on those scales. US nurse researcher Mecca Cranley, using Rubin's work on the development of the maternal role in pregnancy, created the MFAS in 1981, while John T. Condon, a psychiatrist in Australia, developed the MAAS in 1993.

Though the scales have substantial similarities, they have slightly different conceptualizations of attachment. Cranley defined maternal-fetal attachment as "the extent to which women engage in behaviors that represent an affiliation and interaction with their unborn child" (1981, 282). She used a subscale to measure this attachment, which includes interacting with and ascribing "characteristics and intentions" to the fetus. It also includes "differentiation of self from the fetus" as well as "role-taking" and "giving of self" (282). Condon acknowledged that there is not wide agreement on how to define attachment and chose to define it broadly as "an 'emotional tie' or 'psychological bond' to a specific object" $(1993,167)$. Condon's instrument is based on the theoretical construct that the "core subjective experience of attachment or 'love'" has five components: "disposition to know, disposition to be with (\& interact with), disposition to avoid separation or loss, disposition to protect, [and] disposition to gratify needs" (170). These dispositions in turn lead to certain attachment behaviors, such as "information seeking, proximity seeking, protective/ safeguarding, pleasing, [and] gratifying altruistically" (170).

Despite differences, each scale positively values preoccupation with the fetus. Cranley's questions measure the frequency of a pregnant woman's thoughts that correlate with her subscale, with higher frequency indicating stronger attachment. Condon provides a more indepth discussion of preoccupation and understands healthy attachment as that which has both a positive quality and a high level of preoccupation or "time in 'Attachment Mode'" (1993, 181). Condon's MAAS asks pregnant individuals to rate the frequency of their thoughts about and talking to the fetus as well as the strength of their "desire to read or get information about [the] foetus" (178). The higher the frequency or strength of feeling the better, with the caveat that healthy preoccupation is distinct from "anxious, ambivalent or affectless preoccupation, characterized by ambivalent or detached quality of attachment and high preoccupation" (1993, 181). In fact, he explicitly opposes "positive" quality of attachment to an "ambivalent or detached" quality (1993, 181). Condon's breakdown illustrates a microanalysis and micromanagement of women's emotions. Not only should women be preoccupied with the fetus, they must not express anxiety-despite perhaps having good reason to be anxious-and they must not be ambivalent.

In addition to measuring women's frequency of thoughts and level of preoccupation with the fetus, both the MFAS and MAAS correlate healthy attachment with significant lifestyle 
changes during pregnancy. For example, Condon's scale measures how frequently a pregnant woman is concerned about her diet $(1993,178)$. Another researcher adapted questions from Cranley's instrument that include: "I give up doing certain things because I want to help my baby" and "I feel all the trouble of being pregnant is worth it" (Pretorius 2006, 1418). These questions reflect an assumption that pregnancy is a special event that necessitates significant changes in behavior and individual consumption, rather than a commonplace, normal experience that can fit into the fabric of one's existing lifestyle. They also illustrate the heightened medical attention to women's consumption during pregnancy as the locus of risk to the fetus. Moreover, self-monitoring and surveillance are presented as a marker of healthy attachment.

These instruments also assume that thinking of the fetus as a unique person characterizes optimal bonding. Questions adapted from Cranley's scale include: "I can almost guess what my baby's personality will be from the way he/she moves, I have decided on a name, ... [and] I refer to my baby by a nickname" (Pretorius 2006, 1418). One example of a question that would measure the quality of a woman's feelings about the fetus on Condon's schema is: "'Over the past week I think of the developing baby mostly as ' . . . 'A real little person inside me with special characteristics'; 'A baby like any other'; 'A human being'; 'A living thing'; 'A thing not yet really alive'" (Sedgmen 2006, 247). These scales thus reflect the belief that it is good for women to view the fetus as a unique person, as the phrase "[a] real little person inside me with special characteristics" illustrates. This is, of course, a contested view of fetuses, especially in the context of abortion politics. Aside from that context, these scales undervalue the views of pregnant people who emphasize the deep connectivity of the fetus to themselves and others. In other words, this question reflects the epistemological priority of the individual under liberal capitalism, which devalues more relational or communal understandings of the self and the fetus, as well as such understandings of the practice of mothering (Collins 1990; West 1988).

Another aspect of the maternal-fetal attachment instruments that Cranley and Condon separately adopted is a focus on visualizing and imagining the fetus. The questionnaire adapted from Cranley includes: "I try to picture what the baby will look like" (Pretorius 2006, 1418). Condon emphasized the importance of projection:

Over the course of a pregnancy, both parents normally acquire an increasingly elaborated internalized representation of the foetus. This comprises a curious admixture of fantasy and reality, the foetus being a recipient par excellence of projection. It is to this internalized image that the emotional tie develops. $(1993,168)$

Condon's questionnaire attempts to measure this projection by asking women to rate where they fall on the following: "Clear/vague mental picture of foetus," "Frequent/infrequent picturing foetus in imagination," and "Frequent/infrequent dreams about baby" (174).

Importantly, the development of the idea of maternal-fetal attachment and its measurement via these instruments, creates a new population: pregnant women with low attachment to their fetuses. A subsequent medical opinion piece discussed below labels this population "sub-optimal" bonders (Campbell 2006a). While the researchers view themselves as simply describing and measuring an independent phenomenon, these practices actually bring 
into existence a new population (Hacking 1986). Their research lays the groundwork for further measuring and management of this population of poor attachers to fetuses. In other words, the instruments themselves help to define poor maternal-fetal attachment as a problem to be addressed. The development of tools to measure attachment solidifies the validity of the thing being measured-that is, the notion that pregnant women can and should bond to their fetuses in a particular way.

Like other aspects of medical science, these instruments are exemplary of biopower, which, according to Michel Foucault, concerns dominion over "living beings" and "life itself" $(1978,142-43)$. Biopower coincides with and is evident in the emergence of the population as a political problem to be managed, partly through human sciences (Foucault 1990). The management of pregnancy, including pre-abortion ultrasound mandates, is a clear example of the management of life itself (Rodrigues 2014). In creating a concept of, and an instrument to measure, maternal-fetal attachment, medical researchers use affect to manage and exert power over life. Scientists measuring attachment also utilize already common population variables such as "state of health" and "patterns of diet" (Foucault 1978, 25). For example, Condon suggested that low scores on the questionnaire could be correlated to membership in an "at-risk group" of people "less likely to abstain from smoking and alcohol use during pregnancy" and "more likely to suffer pathological grief in the event of foetal loss" $(1993,182)$. Condon thus forged a potential link between a particular affect and a range of emotional and behavioral variables. As such, examining the research on clinical uses of ultrasound can illuminate how the normal prenatal bonder is defined and how ultrasound is viewed as a potential fix for the abnormal, or even just "sub-optimal," bonder.

The research examined here illustrates the importance of affect in this exercise of power over life. This medical discourse treats intense attachment as a self-evident, universal good. Just as Ahmed argues that "[w]hen happiness is assumed to be a self-evident good, then it becomes evidence of the good" $(2010,13)$, we could say that when attachment is assumed to be a selfevident good, then it becomes evidence of the good. I also argue, following Ahmed, that attachment, like happiness, "is used to redescribe social norms as social goods" (2). In this instance, the social norm and thus social good is intense attachment. Another element of this research is that, like the science of happiness that Ahmed critiques, the science of maternal-fetal bonding relies on self-reporting your feelings, as though such feelings are clear and straightforward. Because the feelings are reported "through categories that are value laden" (5), critically evaluating these measurements must involve interrogating the values they presume. Here they reveal that negative or ambivalent feelings about pregnancy are not only less valued but, as elaborated in the next section, are viewed as a problem with medical consequences.

Cranley's and Condon's research leaves somewhat open the question of what should be done about poor attachment. Each suggests that lack of social and familial support, as well as psychological issues like depression may be related to poor attachment (Condon 1993, 182; Cranley 1981, 284). Yet the researchers who employ questionnaires to examine ultrasound's effect on bonding tend to downplay the potential link between social support and bonding. Many of them instead focus on how to use techno-medical means to encourage bonding as though simply 
showing a woman an image of a fetus can help address a problem like depression or lack of social support. By offering ways to measure attachment, Cranley's and Condon's work nonetheless lays important groundwork for individualizing the cause and treatment for a whole range of threats to infant well-being. Making mother love real through measurement opens up the potential to control and manipulate pregnancy in new ways. In Rothman's terms, these scales extend the ideology of technology to the emotional realm. Emotions become things that the medical profession can measure, study, and try to optimize. In particular, it becomes possible to study, with seeming rigor, how ultrasound affects maternal-fetal bonding.

\section{Ultrasound as Emotional and Behavioral Therapy}

[T] he effect of this new prenatal technology [of ultrasound] on the emotional and moral component underlying parental acceptance or rejection of the fetus constitutes a natural social experiment. Parental recognition of the fetal form is a fundamental element in the later parent-child bond. Therefore, a social change with potentially important but perhaps largely unrecognized consequences will gradually unfold from beneath the medical canopy. -John C. Fletcher and Mark I. Evans'

\section{Background and Methods}

The turn toward viewing ultrasound as a way to alter women's emotions toward fetuses coincided with the development of Cranley's instrument. These attachment instruments emphasize the very visualization of the fetus that ultrasound enables and that Western science privileges. This visualization is in turn central to the medical management of pregnancy as reflected in the above quote. The quote is from a 1983 research note that is the only medical text that the 2011 AUL model legislation cites for its claim "that women feel bonded to their children after seeing them on the ultrasound screen" (AUL 2011). The citation in the AUL guide leaves the impression that the piece is a peer-reviewed study from the New England Journal of Medicine. However, the paper is an observational note in which the authors relate two anecdotes of women who said that having an ultrasound helped them bond to their fetuses and decide against having an abortion. The authors conclude simply that ultrasound's role in facilitating bonding should be studied, which subsequent studies in fact did.

As mentioned above, in the 1980s researchers discovered the topic of women's reaction to fetal sonograms. This research is almost exclusively conducted on women who are seeking prenatal care and thus likely not contemplating abortion. Although the English-language studies in this area are conducted in a variety of countries mostly in the Global North, researchers tend not to discuss the cultural and political context in which they are researching. I gathered the

"From "Maternal Bonding in Early Fetal Ultrasound Examinations" (1983) New England Journal of Medicine 308:392-93.

? See Taylor (2008) for an extended discussion of the Fletcher and Evans note. 
archive for the following discourse analysis using keyword searches for "ultrasound" combined with variations of "maternal-fetal attachment" such as "maternal-fetal bonding" and "prenatal attachment" in the PubMed database. A full review of all of the research on ultrasound's emotional effects during pregnancy is beyond the scope of this article. For example, I omitted all studies that concerned bonding and diagnosis of prenatal abnormality, which is the basis of a separate ongoing project. In addition, I included only articles that either were primarily focused on studying the effect of ultrasound on bonding or in which bonding or attachment served as a major interpretive frame in understanding the results of an ultrasound study. I also limited my archive to studies that used self-assessment questionnaires as a primary method, and my analysis focuses on how the scales examined in the previous section are used in the limited research on ultrasound and bonding. ${ }^{.}$My research also turned up a number of influential notes and editorials in medical journals as well as a handful of articles that didn't strictly meet my search criteria but were frequently cited in the studies under review. Many of these texts further substantiate important points from my analysis of the research articles. The editorials, in which researchers can offer less-restrained commentary than in a peer-reviewed study, both corroborate my interpretation of the studies and offer additional illuminating context. The studies identified in the ways indicated above as well as a broader reading of related medical texts thus inform my analysis of the limited research in this area.

My search yielded a total of 15 research articles published between 1980 and 2013. Within this time range, there were two main peaks of activity. Six articles were published in the 1980s and seven were published between 2005 and 2007. The outliers were a single article published in the late 1990s and one article published in 2013. The articles published in these different periods of activity have slightly different characteristics. The earlier studies were focused on 2D ultrasound while many of the later articles examined the effect of more recently developed technologies like $4 \mathrm{D}$ ultrasound-which represents the fetus in three spatial dimensions and moving in real time-on maternal-fetal bonding. Only one of the earlier studies used a scale discussed in the previous section (the MFAS), whereas all but two of the studies published in 2005 or later used the MFAS or MAAS (two used the MFAS while four used the MAAS). The rest of the articles used their own questionnaires, though they often reflected understandings of attachment similar to those expressed in the MAAS and MFAS. Another difference between the two periods is that the studies in the 1980s were published in a variety of journals, including journals specializing in nursing, psychiatry, and psychology. In contrast, most of the articles published between 2005 and 2007 were published in two specialty ultrasound journals: The Journal of Ultrasound in Medicine (JUM) and Ultrasound in Obstetrics and Gynecology (UOG), which

s This meant that I excluded three articles (two of which were published in 2016), all from Scandinavian countries, that employed more detailed and intensive qualitative methodologies such as grounded theory and in-depth interview. These studies also offered nuanced discussion of the role of ultrasound and saw it as having therapeutic potential as part of a holistic treatment plan. They thus differed in many ways from the literature reviewed here and illustrate a distinct way to approach fetal ultrasound in clinical practice.

' Only four of the studies examined the effect of ultrasound on fathers' bonding as well as the pregnant individuals so I leave out here an investigation of bonding for fathers. 
published their first issues in 1982 and 1991, respectively. Despite these differences, the frames of the articles in the two periods were quite similar.

It is worth noting that UOG stood out as an important locus of this research. UOG has published not only three research articles on the effect of ultrasound on maternal-fetal bonding but also two editorials since 2002. Its associated "professional membership association and charity," International Society for Ultrasound in Obstetrics and Gynecology, is the leading professional organization for the field. Moreover, the journal's founding editor and the society's first president, Stuart Campbell, is a prominent obstetrician of fetal ultrasound generally and maternal-fetal bonding and ultrasound specifically. Campbell himself penned the two editorials (2002; 2006a) in the journal opining the promise of ultrasound to enhance maternal bonding. He also drew on his experiences of seeing sonograms and witnessing parents' reactions to them to argue publicly for lowering the gestational age at which a woman can obtain an abortion for social reasons in the UK to 18 weeks (Campbell 2006b). Although an in-depth study of the links between anti-abortion politics and this area of medical study is beyond the scope of this article, Campbell's public commentary and the assumptions of the AUL ultrasound mandates reveal what pro-life advocates may see as at stake in this field of study.

My interpretation of the studies published in UOG and elsewhere revealed three main framings of the research that uses self-assessment to measure the effects of seeing a fetal sonogram on a pregnant woman's attachment to her fetus. These characteristics are: by offering visualization of the fetus, ultrasound enables women to understand the reality and personhood of the fetus; understanding this about the fetus positively influences maternal attachment; and, finally, enhanced attachment is likely to improve maternal behavior. Although the vast majority of the studies were conducted on obstetrically low-risk populations with little discussion of demographics, several researchers comment that ultrasound may be most useful for "at risk" women. I examine these themes primarily through extended discussion in the following subsection of a representative research study-which includes tracing its claims about attachment and ultrasound-and an editorial. Throughout I make additional references to and discussion of other texts for substantiation.

\section{The Medical Literature}

I begin with the opening paragraph of the primary study I will discuss, a 2006 article by C. F. Zachariah Boukydis and colleagues that used the MFAS to examine the effect of a "standard care" obstetric ultrasound against a more comprehensive "ultrasound consultation":

There is a growing body of evidence that viewing prenatal sonograms increases positive maternal feelings toward the fetus and influences maternal attachment to the fetus at an early stage of pregnancy. Ultrasound examinations are welcomed by most women and contribute to maternal "personification" of the fetus. Prenatal interventions, especially ultrasound examinations, in pregnancies in which there is high psychosocial risk and active substance abuse have the potential to increase maternal-fetal attachment and 
reduce the risk of behaviors that may harm the fetus and compromise the health status of the pregnancy. (Boukydis et al. 2006, 721)

This quote illustrates how sonograms are linked to attachment, to personification, and to maternal behavior change. These three distinct potential effects of sonograms are juxtaposed, suggesting that they are related, though no clear causal link is made. Note that the concluding sentence is rather weak, merely suggesting that sonograms have potential for facilitating attachment in certain high-risk populations.

Examining the few studies that Boukydis et al. cite for their claim that ultrasound "influences" attachment will allow me to explore other studies in my archive and demonstrate how these studies relate to one another. The first reference is to a literature review published in 1990 that emphasized the limitations of existing studies (Lumley 1990). The other two references are to studies that came up in my searches. The first-a 2005 study in UOG that examined the effect of 2D ultrasound compared with 3D ultrasound-concluded that the latter, "by making it possible to better visualize the baby, may have the potential benefit of increasing the bonding of mothers to their expected newborns and strengthening the support system for their families" (Ji et al. 2005, 476). In addition to questions that asked women to assess "how helpful" the sonogram was in terms of "knowing the baby was healthy, in making the parents see the fetus as real and in feeling a closer relationship with the baby," the study's data included women's reports of the number of people to which they showed their sonograms (474). Unsurprisingly, they found that those with a 3D image, which is more novel, showed the images to more people. Moreover, the authors note the limitations of their study because they did not use a validated attachment instrument such as the MFAS. These researchers also naturalized the desire to see the fetus, writing that "parents have a natural desire to see and know their baby before birth" (475). In doing so, they ignore that this supposedly natural desire is only a possibility after the technological innovation of ultrasound. In taking for granted this desire and the naturalness of maternal-fetal bonding, these researchers ignore how these "natural" phenomena may be socially and technologically constructed.

The second study that Boukydis et al. cite for the claim about ultrasound's influence on attachment was published in 2005 and used the MAAS to compare the effect of 2D ultrasound to 2D plus $4 \mathrm{D}$ ultrasound. In contrast to the other study, however, this study concluded that "addition of $4 \mathrm{D}$ ultrasound does not change significantly the perception that women have of their baby nor their antenatal emotional attachment compared with conventional 2D ultrasound" (Rustico et al. 2005, 468). The referenced study nonetheless makes use of the common themes of this research, noting in the introduction that "visualization of the fetus by ultrasound might arouse emotions capable of triggering prenatal attachment, which makes the fetus into a person and influences maternal compliance and lifestyle" (Rustico et al. 2005, 469). This quote-as well as the two studies I found that go so far as to describe the fetus as "autonomous" (Reading et al. 1984; Colucciello 1998) - illustrate that fetal personhood is accepted rather uncritically as the way to understand the fetus at least in part because such a view leads to, or is correlated with, the right affective orientation toward the fetus and pregnancy. 
Like the studies it references, the Boukydis study itself is a good example of the limited findings that characterize this body of research. Because the researchers studied only the effect of routine ultrasound against ultrasound with an extended consultation, they merely conclude that ultrasound consultation-not ultrasound itself-positively changed maternal-fetal attachment scores. Moreover, the researchers note that the consultation significantly affects the "attributing fetal characteristics" subscale of the MFAS. Yet it is hardly surprising that an ultrasound with an extended consultation increases this. As the very reviewer that Bouykidis et al. cite for the claim that ultrasound influences attachment explained about the MFAS:

items in the subscales "attributing characteristics and intention to the fetus" and "interaction with the fetus" may be directly affected by the ultrasound information and the way it is given. The MFA score would be increased by the very process under investigation ... (Lumley 1990, 215)

As a whole, the studies I reviewed are premised on the idea that affect can and should be changed even when researchers' findings did not substantiate the notion that ultrasound itself changed pregnant women's affect. As Ahmed gleans from John Locke's idea of “correctible taste," "[t]he very possibility that we can affect our affections by action, or through will or reason, becomes the basis of an ethical imperative" $(2010,36)$. The medical literature studied here shows how this ethical imperative is translated into seemingly neutral medical science. This notion that our affect can be changed undergirds the use of technical imagery to bring about an affective reorientation. This literature presents lack of proper feeling toward the fetus as something that can be corrected through technology. Instead of examining deeply why a certain feeling toward the fetus exists, much of the literature I reviewed suggests that ambivalence about pregnancy-which may result from things such as feminist consciousness or one's particular social and economic circumstance-is a problem. Recall that the MAAS, in fact, opposes ambivalent attachment with positive attachment so that studies that use the MAAS explicitly replicate the characterization of ambivalence as a problem.

In 2006, Campbell published an editorial in UOG. His piece, which called for more research on the effect of $4 \mathrm{D}$ ultrasound for bonding, described maternal-fetal attachment as a "natural phenomenon" and "an essential process in the development of good and loving motherly behavior" (2006a, 243). A corollary of this idea is that sub-optimal bonding is an attribute of bad mothering and may lead to poor fetal and neonatal outcomes. Certain risks to infant well-being, then, become located in women's emotional state. Campbell characterizes the population that fails to develop this natural attachment as "sub-optimal" bonders. As a prominent advocate for the theory that ultrasound promotes bonding, Campbell's editorializing on the process is significant. He clearly wants ultrasound to play an important role in bonding throughout pregnancy. He writes: "It is now widely accepted that the early routine scans at 12 and 20 weeks are the main factors involved in initiating this bonding process, although disappointingly, ultrasound has not been shown to intensify this process in the 3rd trimester" (2006a, 243). 
Campbell nonetheless remains optimistic and the point of his editorial is to call for more research into the potential for the more lifelike $4 \mathrm{D}$ ultrasound to enhance bonding later in pregnancy: "It is the visible humanity of the fetus at this stage, the baby-like facial expressions, and the sucking, grasping and other movements that I believe could trigger a surge in bonding in the last 16 weeks of pregnancy" (243). Campbell and other researchers tend to think that the better the technology, the more baby-like the picture will be. The appeal to the realism of the image obscures that viewing the fetus as akin to a baby is a value judgment about the meaning of pregnancy. Moreover, Campbell hopes that the effect of ultrasound on bonding will become more central to obstetrical practice and believes this will happen if a concrete effect on behavior is demonstrated. He concludes that future research should "target the cohort of women who are sub-optimal bonders and should assess the impact of the scan on tangible health-behavior benefits" (244).

The slippage from emotions to behavior here and throughout this research reflects a growing concern since the 1970s on women's consumption during pregnancy. Elizabeth Armstrong has written about how, despite uncertain evidentiary support, fetal alcohol syndrome (FAS) was "discovered" in 1973. As she argues, FAS "chang[ed] our perceptions of risk and responsibility" and thereby reworked the understanding of the relationship between a pregnant woman and her fetus. For her, the critical change was "from thinking of the woman and the fetus as a single entity to thinking of the woman and the fetus as two separate individuals" $(2008,9)$. This is the precursor for the notion of maternal-fetal conflict. Only if the fetus is thought of as an individual, separated from the pregnant individual, is it possible to conceive of a conflict between the interests or well-being of pregnant woman and fetus. In turn, then, only in the separation of fetus from pregnant woman is it possible to think of attachment between them. In other words, anxiety over maternal-fetal attachment can arise only when the notion of the fetus and woman as one entity deteriorates. The technology of ultrasound has played a key role in bringing about the separation of the fetus from woman.

Although some researchers note the limitations of ultrasound to correct sub-optimal bonding, studies on ultrasound's ability to enhance maternal-fetal attachment still tend to frame it as an emotional therapy. After all, the studies analyzed here all examine the effect of ultrasound on pregnant women's self-assessment of their feelings about the fetus and pregnancy before and after a particular kind of ultrasound scan. Furthermore, the studies overwhelmingly posit that in some form ultrasound can enhance attachment and thus improve prenatal and neonatal health. Pregnant women's emotions thus become a central focus of risk for a very generalized understanding of fetal and baby well-being. Altering women's affect is viewed as a way to get women to comply with health recommendations and change the fetal environment. In many ways, the main characteristics of fetal ultrasound research echo what the MFAS and MAAS assume. What is distinct is the focus on ultrasound to alter the strength of a woman's attachment. Additionally, since so many of the studies take for granted that questionnaires can accurately and unproblematically measure the independent quality of maternal-fetal attachment, this body of research replicates and reinforces the shortcomings of those questionnaires detailed above. 


\section{Conclusion}

This article brings affect studies to STS and extends Ahmed's insights more directly to reproduction and technology. Though much of The Promise of Happiness concentrates on the family, Ahmed does not fully flesh out the importance of reproduction and pregnancy to the affective community that shares the orientation to the family as good and constructs the family as a happy object. In the literature on ultrasound and maternal-fetal bonding, the bond stands in for the feeling that you have what should make you happy. The bond, as a reflection of the child, is a sort of promise of happiness-in particular, the happiness of motherhood. To be conscious of the unhappiness that might attend motherhood and thus to have negative or ambivalent feelings about pregnancy and the fetus is to be rendered a problem. Moreover, Ahmed notes that "some bodies more than others will bear the promise of happiness" (2010, 45), though she never discusses the fetal body. My analysis here supplements Ahmed's work to illuminate, through a unique archive, the construction of the fetal body as an object of happiness.

Ahmed's work reveals further that affect is used to distract from larger systematic injustices and in some circumstances to make the origin of bad feeling, not injustice, but feminist consciousness. In her words, although feminists may be "read as destroying something that is thought of by others not only as being good but as the cause of happiness," feminists can instead be understood as "expos[ing] the bad feelings that get hidden, displaced, or negated under public signs of joy" (65). As such, ambivalence about pregnancy could reflect a feminist consciousness. This article shows how medicalization can reify a view of the fetus as an object of love and happiness such that a particular kind of maternal-fetal bond is held up both as good and medically indicated.

Moreover, as other STS researchers have done, this article asks us to question that which is taken as a universal good-here, a particular kind of mother love-rather than simply asking for more inclusive access to that good (see Reardon 2013). In fact, the maternal-fetal bonding selfassessment scales described above reflect a valorization of the intensive mothering ideal that many scholars have critiqued for reflecting white middle-class norms (Collins 1990; Roberts 1998). In Evelyn Nakano Glenn's words, the ideology of good mothering places responsibility "almost exclusively on one woman (the biological mother), for whom it constitutes the primary if not sole mission during the child's formative years" and is "derived from the situation of the white, American, middle class" yet presented as universal $(1994,3)$. Importantly, the ideal of intensive mothering (Hays 1998) is not equally available to all parents, nor is it universally desirable. For example, for many low-income African-American families, taking care of children is a collective community responsibility, not an intensive individual endeavor (Stack and Burton 1994). And, as bell hooks writes, "the idea of an individual having sole responsibility for childrearing is the most unusual pattern of parenting in the world, one that has proved to be unsuccessful because it isolates children and parents from society" $(1984,143)$. This link suggests avenues for future research to explore the racialized and classed aspects of research on maternalfetal bonding and the clinical practice of ultrasound. Given the ubiquity of prenatal ultrasound, as well as the increasing legal and political attention to prenatal bonding in the US, this would be an important line of inquiry. 


\section{Author Biography}

Jennifer Denbow is an Assistant Professor of Political Science at Cal Poly, San Luis Obispo. Her work combines critical theory and transdisciplinary feminist studies to investigate contemporary reproductive law, technology, and politics. She is the author of Governed through Choice: Autonomy, Technology, and the Politics of Reproduction (NYU Press, 2015). Her work has also appeared in a variety of journals, including Signs, Constellations, and Feminist Legal Studies.

\section{Acknowledgements}

I am grateful for the critical engagement of a number of commentators and collaborators at various stages of this project. I would like to recognize the anonymous reviewers and editors of ESTS for their astute and understanding commentary. Thank you also to James Doucet-Battle for his insightful feedback at the American Ethnological Society Conference and to the organizers and participants of the Race, Reproduction, Risk panels at that conference. I would also like to acknowledge my collaboration with Nick Lally on a related project that informed the present work. Finally, I am thankful for the helpful research assistance of Nestor Veloz-Passalacqua at an early stage of this project and for the invaluable work of Nicole Chen at a later stage.

\section{References}

Ahmed, S. 2010. The Promise of Happiness. Durham, NC: Duke University Press.

Americans United for Life (AUL). 2011. “Women's Ultrasound Right to Know Act." Accessed 20 February 2018: http:/ / www.aul.org/ defending-life-2011-model-legislation-abortion/

Armstrong, E. 2008. Conceiving Risk, Bearing Responsibility: Fetal Alcohol Syndrome and the Diagnosis of Moral Disorder. Baltimore, MD: Johns Hopkins University Press.

Boukydis, C.F.Z., M.C. Treadwell, V. Delaney-Black, et al. 2006. “Women's Responses to Ultrasound Examinations During Routine Screens in an Obstetric Clinic." Journal of Ultrasound in Medicine 25:721-726.

Brandon, A., S. Pitts, W. Denton, et al. 2009. "A history of the theory of prenatal attachment." Journal of Prenatal and Perinatal Psychology and Health 23(4):201-222.

Campbell, S. 2002. "4D, or not 4D: that is the question." Ultrasound in Obstetrics and Gynecology 19:1-4.

Campbell, S. 2006a. "4D and prenatal bonding: still more questions than answers." Ultrasound in Obstetrics and Gynecology 27:243-44.

Campbell, S. 2006b. "Don't tear a smiling fetus from the womb." The Telegraph. Accessed 20 February 2018. http://www.telegraph.co.uk/comment/personal-view/3632855/Donttear-a-smiling-foetus-from-the-womb.html

Casper, M. 1998. The Making of the Unborn Patient: A Social Anatomy of Fetal Surgery. New Brunswick, NJ: Rutgers University Press.

Collins, P. 1990. Black Feminist Thought. New York: Routledge. 
Colucciello, M. L. 1998. "Pregnant Adolescents' Perceptions of their Babies before and after Realtime Ultrasound." Journal of Psychosocial Nursing and Mental Health Services 36(11): 12-19.

Condon, J. 1993. "The assessment of antenatal emotional attachment: Development of a questionnaire instrument." British Journal of Medical Psychology 66:167-83.

Cott, N. 1977. The Bonds of Womanhood. New Haven, CT: Yale University Press.

Cranley, M. S. 1981. "Development of a Tool for the Measurement of Maternal Attachment During Pregnancy." Nursing Research 30(5):281-84.

Cunen, N., J. Jomeen, R. Xuereb, et al. 2016. "A narrative review of interventions addressing the parental-fetal relationship." Women and Birth. http:// dx.doi.org/10.1016/j.wombi.2016.11.005

Degler, C. 1984. At Odds: Women and Family from the Revolution to the Present. New York: Oxford University Press.

Denbow, J. 2015. Governed through Choice: Autonomy, Technology, and the Politics of Reproduction. New York: NYU Press.

Duden, B. 1993. Disembodying Women: Perspectives on Pregnancy and the Unborn. Cambridge, MA: Harvard University Press.

Epstein, S. 2007. Inclusion: The Politics of Difference in Medical Research. Chicago, IL: University of Chicago Press.

Eyer, D. 1992. Mother-infant bonding: A scientific fiction. New Haven, CT: Yale University Press.

Foucault, M. 1978. The History of Sexuality: Volume I. New York: Vintage.

Foucault, M. 1990. “On power." In: Politics, philosophy, culture: Interviews and other writings, 19771984. New York: Routledge.

Fujimura, J. 2006. "Sex Genes: A Critical Sociomaterial Approach to the Politics and Molecular Genetics of Sex Determination." Signs: Journal of Women in Culture and Society 32(1):49-82.

Franklin, S. 1991. "Fetal fascinations: New dimensions to the medical-scientific construction of fetal personhood." In: Off-Centre: Feminism and Cultural Studies, edited by S. Franklin C. Lury, and J. Stacey, 190-205. New York: Harpercollins Academic.

Glenn, E. 1994. "Social Constructions of Mothering." In: Mothering: Ideology, Experience, and Agency, edited by E. Glenn, G. Chang, and L. Forcey. New York: Routledge.

Guttmacher Institute. 2018. "State Laws and Policies: Requirements for Ultrasound." Accessed 20 February 2018. https://www.guttmacher.org/state-policy/explore/requirementsultrasound

Hacking, I. 1986. "Making Up People." In: Reconstructing Individualism: Automony, Individuality, and the Self in Western Thought, edited by T. Heller, M. Sosna, and D. Wellberg. Stanford, CA: Stanford University Press.

Haraway, D. 1988. "Situated Knowledges: The Science Question in Feminism and the Privilege of Partial Perspective." Feminist Studies 14(3):575.

Hays, S. 1998. The Cultural Contradictions of Mothering. New Haven, CT: Yale University Press. hooks, b. 1984. Feminist Theory: From Margin to Center. Boston, MA: South End Press. 
Ji, E., D. Pretorius, R. Newton, et al. 2005. "Effects of ultrasound on maternal-fetal bonding: a comparison of two- and three-dimensional imaging." Ultrasound in Obstetrics $\mathcal{E}$ Gynecology 25(5):473-477. doi:10.1002/uog.1896.

Joyce, K. 2005. "Appealing Images: Magnetic Resonance Imaging and the Production of Authoritative Knowledge." Social Studies of Science 35(3):437-462.

Kukla, R. 2008. "Measuring Mothering." International Journal of Feminist Approaches to Bioethics 1(1):67-90.

Lappé, M. 2016. “The maternal body as environment in autism science." Social Studies of Science 46(5):675-700.

Lumley, J. 1990. “Through a Glass Darkly: Ultrasound and Prenatal Bonding.” Birth 17(4):214217.

Mitchell, L. 2001. Baby's First Picture. Toronto: University of Toronto Press.

Morgan, L. 2009. Icons of Life: A Cultural History of Human Embryos. Berkeley, CA: University of California Press.

Newman, K. 1996. Fetal positions: Individualism, science, visuality. Stanford, CA: Stanford University Press.

Oakley, A. 1984. The Captured Womb: A history of the medical care of pregnant women. Oxford, UK: Blackwell Publisher.

Oaks, L. 2000. "Smoke-filled wombs and fragile fetuses: The social politics of fetal representation." Signs: Journal of Women in Culture and Society 26(1):63-108.

Palmer, J. 2009. "The placental body in 4D: Everyday practices of non-diagnostic sonography." Feminist Review 93(1):64-80.

Petchesky, R. 1987. "Fetal Images: The Power of Visual Culture in the Politics of Reproduction." Feminist Studies 13(2):263.

Pretorius, D., et al. 2006. "Preexamination and postexamination assessment of parental-fetal bonding in patients undergoing 3-/4-dimensional obstetric ultrasonography." The Journal of Ultrasound in Medicine 25:1411-1421.

Rapp, R. 2000. Testing Women, Testing the Fetus: The Social Impact of Amniocentesis in America. New York: Routledge.

Reading, A. E., et al. 1984. "Psychological Changes Over the Course of Pregnancy: A Study of Attitudes Toward the Fetus/Neonate." Health Psychology 3(3):211-221.

Reardon, J. 2013. "On the Emergence of Science and Justice." Science, Technology, and Human Values 38(2):176-200.

Richardson, S. 2015. "Maternal Bodies in the Postgenomic Order: Gender and the Explanatory Landscape of Epigenetics." In: Postgenomics: Perspectives on Biology after the Genome, edited by S. Richardson and H. Stevens. Durham, NC: Duke University Press.

Roberts, D. 1998. Killing the black body: Race, reproduction, and the meaning of liberty. New York: Vintage.

Roberts, J. 2016. The Visualised Foetus. New York: Routledge. 
Roberts, J., F.E. Griffiths, A. Verran, and C. Ayre. 2015. “Why do women seek ultrasound scans from commercial providers during pregnancy?" Sociology of Health and Illness 37(4):594609.

Rodrigues, S. 2014. “A Woman's 'Right to Know'? Forced Ultrasound Measures as an Intervention of Biopower." International Journal of Feminist Approaches to Bioethics. 7(1):5173.

Rothman, B.K. 2000. Recreating Motherhood. New Brunswick, NJ: Rutgers University Press.

Rubin, R. 1967a. "Attainment of the Maternal Role. Part I. Processes." Nursing Research. 16(3): 237-245.

Rubin, R. 1967b. "Attainment of the Maternal Role. Part II. Models and Referrants." Nursing Research. 16(4):342-346.

Rubin, R. 1976. "Maternal Tasks in Pregnancy." Journal of Advanced Nursing. 1(5):367-376.

Ruhl, L. 2002. "Dilemmas of the Will: Uncertainty, Reproduction, and the Rhetoric of Control." Signs: Journal of Women in Culture and Society 27(3):641-663.

Rustico, M.A., C. Mastromatteo, M. Grigio, et al. 2005. "Two-dimensional vs. two- plus fourdimensional ultrasound in pregnancy and the effect on maternal emotional status: A randomized study." Ultrasound in Obstetrics and Gynecology 25:468-472.

Sedgmen, B., M. McMahon, D. Cairns, et al. 2006. “The impact of two-dimensional versus threedimensional ultrasound exposure on maternal-fetal attachment and maternal health behavior in pregnancy." Ultrasound in Obstetrics \& Gynecology 27(3):245-251. doi:10.1002/uog.2703.

Shrage, L. 2002. "From Reproductive Rights to Reproductive Barbie: Post-Porn Modernism and Abortion." Feminist Studies 28(1):61-93.

Sioda, T. 1984. "Psychological effects of cardiotocographic and ultrasonographic examinations in pregnancy and labour on the mother. Part 1 . The significance of cardiotocographic and ultrasonographic examinations for the development of maternal bonding." Ginekologia Polska 55(9):653-660.

Stack, C.B. and L.M. Burton. 1994. "Kinscripts: Reflections on Family, Generation, and Culture." In Mothering: Ideology, Experience, and Agency, edited by E.N. Glenn, G. Chang, and L.R. Forcey. New York: Routledge.

Taylor, J. 2002. "A Fetish is Born: Sonographers and the Making of the Public Fetus." In: Consuming Motherhood, edited by J. Taylor, L. Layne, and D. Wozniak. New Brunswick, NJ: Rutgers University Press.

Taylor, J. 2008. The Public Life of the Fetal Sonogram: Technology, Consumption, and the Politics of Reproduction. Brunswick, NJ: Rutgers University Press.

Vicedo, M. 2013. The nature and nurture of love: From imprinting to attachment in cold war America. Chicago, IL: Chicago University Press.

Waggoner, M.R. 2013. "Motherhood Preconceived: The Emergence of the Preconception Health and Health Care Initiative." Journal of Health Politics, Policy and Law 38(2):345-371.

Waggoner, M.R. 2015. "Cultivating the Maternal Future: Public Health and the Pre-Pregnant Self." Signs: Journal of Women in Culture and Society 40(4):939-962. 
West, R. 1988. “Jurisprudence and Gender." University of Chicago Law Review. 55(1):1-72.

Wolf, J.B. 2011. Is Breast Best? Taking on the Breastfeeding Experts and the New High Stakes of Motherhood. New York: NYU Press. 